\title{
Etiopathogenesis of dermatitis herpetiformis
}

\author{
Joanna Rybak-d’Obyrn, Waldemar Placek \\ Department of Dermatology, Sexually Transmitted Diseases and Clinical Immunology, University of Warmia and Mazury, Olsztyn, Poland \\ Adv Dermatol Allergol 2022; XXXIX (1): 1-6 \\ DOI: https://doi.org/10.5114/ada.2020.101637
}

\begin{abstract}
Dermatitis herpetiformis is a rare chronic, autoimmune bullous disease linked to gluten sensitivity with intense pruritus and characteristic skin eruptions. Etiopathogenesis is complex and not fully understood. It is currently considered to be a specific cutaneous manifestation of celiac disease. Genetic, environmental and immunological factors influence both conditions. Exposure to gluten is the starting point of an inflammatory cascade leading to the formation of circulating IgA antibodies against tissue transglutaminase and skin immune IgA deposition followed by skin lesions. Binding of the immune complex deposits of IgA transglutaminases and epidermal antibodies with enzymes in the papillary dermis stimulates complement activation, neutrophil influx, proinflammatory cytokine release and overproduction of matrix metalloproteinases. We have collected current knowledge of the pathogenesis of dermatitis herpetiformis.
\end{abstract}

Key words: dermatitis herpetiformis, transglutaminases, vesicobullous, skin diseases.

\section{Introduction}

Dermatitis herpetiformis or Duhring's disease $(\mathrm{DH})$ is a rare, chronic, subepidermal bullous disease, with autoimmunity to transglutaminases, characterized by a chronic relapsing course, pruritic polymorphic lesions and typical histopathological and immunopathological findings. DH etiopathogenesis is complex and only partly understood. Gluten exposure triggers an inflammatory cascade leading to the formation of circulating IgA antibodies to tissue transglutaminase. Genetic predisposition and environmental factors also influence the disease. Characteristic papulovesicular eruption on the skin is associated with severe itching and burning. Diagnosis of the disease is determined by a direct immunofluorescence of the presence of granular IgA deposits in the skin papillae and/or along the cutaneous-epidermal border. It is currently considered to be a specific cutaneous manifestation of celiac disease (CD). Both conditions share the same HLA haplotypes, are mediated by the IgA class of autoantibodies, react with the transglutaminases and improve on gluten-free diet $[1,2]$.

\section{Genetic background}

$\mathrm{DH}$ and $\mathrm{CD}$ are associated with specific human leucocyte antigen (HLA) class II: HLA-DQ2 and HLA-DR3 as well as class I: HLA-A1 and HLA-B8. HLA-DR2 antigen is present only in $\mathrm{DH}[1,2]$. Among HLA-DQ2, DQB1*0201, $D Q A 1^{*} 0501$ alleles are relevant for both diseases, and for HLA-DR3, DRB1*0301 allele, which probably protects, similar as HLA-DR2, against the development of enteropathy in $\mathrm{DH}$ patients $[3,4]$. Villous atrophy correlates with the presence of HLA-B8, which is more common in patients with enteropathy [5]. It is estimated that in $\mathrm{DH}$ or CD, antigens occur in almost all patients: HLA-B8 in 58-90\%, HLA-DR3 in 88-95\% and HLA-DQ2 in 95-100\% compared to healthy people $(21 \%, 23-31 \%, 40-41 \%$, respectively) [6]. In DH, the frequency of HLA-DR2 is estimated to be $57 \%$ compared to the general population $(31 \%)[4,5,7]$. There are also differences depending on the population; there is no HLA-B8, -DR3 and -DQ2 haplotype in Japan but HLA-DR9 is present, which is associated with the fact that $\mathrm{DH}$ hardly ever occurs there and it is not accompanied by enteropathy, and CD is seen very rarely [8].

Determination of these HLA antigens in healthy people is characterized by very low specificity, responsible only for the predisposition to autoimmune response development [7]. The presence of HLA-DQ2/DQ8 alleles in $\mathrm{DH}$ is characterized by high, and HLA-DQ2 very high sensitivity. It can be used in the diagnosis and screening of first-degree relatives and siblings of patients with $\mathrm{DH}[6$, $7,9,10]$. The absence of HLA-DR3, -DQ2 and -B8 antigens

Address for correspondence: Joanna Rybak-d’Obyrn PhD, Department of Dermatology, Sexually Transmitted Diseases and Clinical Immunology, University of Warmia and Mazury, Olsztyn, Poland, e-mail: jrybakdobyrn@wp.pl Received: 16.12.2019, accepted: 24.06.2020. 
has a high negative predictive value, which allows to rule out $\mathrm{DH}[7,11]$.

\section{Environmental factors}

Environmental trigger factors are represented by the ingestion of gluten, a component of the complex protein mixture contained in wheat flour. Gliadin partially digested in the gut comprising digestion-resistant peptides can be modified by tissue transglutaminase (tTg) that increases their antigenicity. $\alpha$-gliadin plays an important role because tTg reacts with it. It is likely that the tTg is a target for IgA class autoantibody deposition in the small bowel mucosa and anti-gliadin and anti-tTg antibodies are formed in the intestinal mucosa [12]. External or intradermal administration of gluten does not stimulate skin lesions and immunoreactive gluten does not find in the skin [1]. The rash responds to a strict glutenfree diet (GFD) and the symptoms return on gluten challenge. Currently, it is believed that a DH immunopathogenesis starts from latent CD in the intestine with a tTg and possibly an anti-epidermal transglutaminase (eTg) autoantibody response and results in immune complex deposits of high avidity IgA anti-eTg antibodies together with the eTg in the dermal papillae [13]. It is suspected that endocrine (hormonal) and immunologic (viral infections) factors play a role in modulating the inflammatory response in $\mathrm{DH}$. Probably $\mathrm{DH}$ pathogenesis is much more complex than a simple interaction between HLA-DQ antigens and gluten [14].

\section{Immunopathological phenomena in $\mathrm{DH}$}

The presence of granular IgA deposits at dermal papillae in direct immunofluorescence (DIF) is the basis for the diagnosis of $\mathrm{DH}$. Circulating IgA antibodies against endomysium (EmA) and/or transglutaminases are also detected. Histopathological examination of skin lesions shows neutrophilic infiltration leading to the destruction of basement zone proteins, which, together with impaired type IV collagen and laminin, results in a damage to anchor fibres and blistering [9].

The role of tTg and eTg enzymes, the main autoantigens in $\mathrm{DH}$ pathogenesis, is not fully understood. Binding of the immune complex deposits of IgA anti-tTg antibodies with tTg in the papillary dermis stimulates complement activation, neutrophil influx, proinflammatory cytokine release and overproduction of matrix metalloproteinases (MMPs) [15-17]. tTg is released in small amounts by mononuclear cells, fibroblasts and intestinal mucosa cells. In DH, oxidative stress affects fibroblast DNA damage especially after exposure to gliadin peptides [18]. In the digestive tract, tTg reacts with gliadin in two ways. In the intestine, tTg binds to gliadin, and then catalyses the formation of gliadin - gliadin, protein and tTg complexes. Immunogenic complex stimulates the production of IgA antibodies to $\mathrm{tTg}$, which disrupts its physiological function. It is likely that the simultaneous deamidation of gliadin increases the affinity of tTg for antigen presenting cells and as a result, gliadin is recognized by intestinal $T$ cells. Initiating an immune response stimulates Th1 and Th2 cells to increase cytokine secretion, which results in destruction of the intestinal mucosa. Increased Th17 cytokines are also reported in $\mathrm{DH}[19,20]$. Tumour necrosis factor $\alpha$ (TNF- $\alpha)$, produced by Th1, leads to the secretion of MMP1, MMP3 by fibroblasts, and B cell maturation stimulates the production of IgA autoantibodies against gliadin, tTg and cross gliadin-tTg complexes as a result of Th2 stimulation. It is believed that $\mathrm{DH}$ is stimulated mainly by Th2 and CD by Th1 $[1,19]$. In untreated DH patients, a high number of T lymphocytes with the TCR $\gamma / \delta$ + receptor is observed in the jejunum mucosa, and this number is low in healthy people. High density of these lymphocytes in untreated patients after starting GFD decreases to the level found in healthy people. The assessment of the number of T lymphocytes with the TCR $\alpha / \delta$ + receptor seems to be a marker of gluten-dependent diseases [21]. Immune gliadin and tTg complexes in the duodenal mucosa are present in healthy people, but their level is significantly lower [22]. Presence of IgA and tTg is also found in the small intestine of DH and CD patients [1].

It remains unclear why initially common immunological phenomena in the intestine differentiate into skin or intestinal pathology. EmAs are directed against tTg, which is its main autoantigen [23]. In DH, presence of circulating anti-tTg antibodies explain intestinal pathology, and anti-eTg antibodies can explain partly skin lesions [13]. Presence of tTg in the skin of DH patients and healthy people show no differences in the distribution of the enzyme [24]. Immune complexes in the papillary dermis of DH patients contain IgA - eTg and do not contain tTg $[13,25]$. It is thought that in patients who develop $\mathrm{DH}$ in the future, $\mathrm{tTg}$ - gliadin complexes formed in the intestine may initiate production of IgA antibodies with low eTg affinity. After a long gluten provocation antibodies develop, with low affinity for tTg and high for eTg, and then the rash appears. DH should be treated as a sign of skin hypersensitivity to gluten in patients with mild CD who produce anti-eTg antibodies with high avidity and affinity [26]. DH late onset in comparison to CD, which often involves children, could be explained by the epitope spreading phenomenon as a result of an autoimmune response that is provoked by exogenous antigen. Probably this mechanism and/or cross-reactivity between tTg and eTg can lead to eTg autoimmunity and formation of circulating IgA-eTg complexes determines development of $\mathrm{DH}$ in some CD patients. The possible mechanism of a directly gluten-induced eTg autoimmunity could not be also excluded [27]. In the skin, IgA-eTg aggregates have been shown to activate fibrinogen, which can be found at the tips of the papillary dermis in a pattern similar 
to that of IgA-eTg aggregates [14]. Constant presence of immunological deposits in the skin is probably associated with a defective tissue macrophages Fc receptor responsible for their poor removal from the circulation. eTg in the deposits may be of cutaneous origin, and under the influence of injuries (Koebner phenomenon), it is displaced to the papillae, which may explain different location of the active enzyme and that present in the deposits. It is also possible to store immune complexes displaced into the skin after the reaction of IgA anti-eTg antibodies with this antigen in other tissues. It is probably related to the enzymatic activity of eTg, which catalyses the formation of aggregates of these complexes with the skin structures, activation of complement, then the influx of specifically sensitized (possibly in the intestine) neutrophils and the release of proinflammatory cytokines, among others, MMP (stromelysin, gelatinase and matrylin), which leads to efflorescence in places typical for $\mathrm{DH}$ [13]. Activity and expression of eTg are also the reason for the formation of complexes in organs other than the intestine and skin, namely the kidneys (IgA nephropathy) in $\mathrm{CD}$ and $\mathrm{DH}$ patients [28].

The presence of granular or fibrillar IgA deposits at the dermal papillae characteristic for $\mathrm{DH}$ do not occur in people without $\mathrm{DH}$. Accompanying IgM, IgG, C3 and fibrinogen deposits are found, as well as eTg in granular deposits [12]. IgA cutaneous deposits, the IgA1, rarely IgA2 class, are polyclonal, contain kappa and lambda chains, do not contain J fragment. Initially, IgA deposits were thought to bind strongly with microfibrils of the papillary dermis layer or cross-react with microfibrils by FAB fragment, however immunoelectron microscopy studies have not confirmed this theory [29]. The key receptor for IgA is probably CD89, which shows a great expression on neutrophils (as a modulator of their function). It may play a role in the immune response to gluten in $\mathrm{DH}$ [30].

In $\mathrm{DH}$, there is an increased expression of vascular endothelial growth factor (VPF/VEGF) - a marker of vascular permeability [31]. DIF DH skin biopsy analysis, performed by Preisz et al. showed that typical IgA dermal papillae deposits are accompanied by deposits in the walls of vessels in $64 \%$ of cases. They occur most commonly in small skin papillae vessels (92\%), and some are found in vessels located subpapillary and they are deposits of mainly IgA - C3 and IgA. IgG is not found at all. Deposits in vascular walls and peri-intravascular skin papillae structures also contain eTg, C3 - eTg or eTg occur partially and no eTg - IgM deposits are found [32].

Pathogenetic hypotheses of effector mechanisms in $\mathrm{DH}$ suggest that intestinal IgA skin deposits activate the complement via an alternative activation pathway, releasing chemotactic factors and the influx of inflammatory cells. This confirms the presence of the complement C3 component in the same location as IgA as well as the activity of properdin and B factor [33]. Histology shows characteristic micro-abscesses and granular IgA-deposits in papillary dermis while C3-deposition can be found in up to 89\%; no deposits of $\mathrm{C} 1 \mathrm{q}$ were found in $\mathrm{DH}$ biopsies [34]. The C5 component is also detected, and its activation releases the $\mathrm{C} 5 \mathrm{a}$ fraction, which chemotactically acts on neutrophils [33].

The effectiveness of sulfones is explained by inhibition of complement activation, since the presence of C3, accompanying IgA deposits, in the skin is found only in the active phase of the disease, and not in asymptomatic treated DPS patients [33]. There are also studies that deny the effect of DPS on the complement system. The C3 concentration in the serum of DH patients remains within normal limits and is not affected by DPS intake [35]. Co-occurrence of vitronectin and complement C9 is also found in the dermal papillae [36]. Immunological deposits are detected, in similar proportions, both in the skin surrounding the lesions as well as in non-affected skin. It proves that also in healthy skin, depending on the complement activation, an inflammatory process and probably other factors affect the appearance of skin lesions. Potassium iodide, applied orally or topical (as a patch test), causes a rash similar to $\mathrm{DH}$, activating local mechanisms, probably of an immunological nature. The patch test is positive in patients with active disease and in patients on non-restricted GFD, while it is usually negative in patients treated with DPS and in remission. DIF examination of unchanged skin and the patch test site, both in symptomatic and in remission DH patients, showed no difference in the presence, amount and distribution of immunoglobulins, complement and fibrinogen [37]. The mere presence of IgA deposits in the skin is not a sufficient condition for skin lesions. Despite circulating antibodies and skin IgA deposits, no increase in IgA production is observed and total IgA serum levels are not elevated. It is probably associated with impaired IgA utilization due to spleen and reticuloendothelial system abnormalities [38].

Adhesion of neutrophils to the endothelial surface, migration through the vessel wall and accumulation at the site of inflammation are also mediated by selectins and $B$ integrins. This is manifested by increased CD11b expression, decreased $L$ selectin expression and increased ability to bind IgA [39]. This phenomenon begins in the intestine, as a result of a mucosal immune response, predisposing neutrophils to settle in the skin as a response to a trauma-dependent local stimulus. In $\mathrm{DH}$, selectin $\mathrm{E}$ has strong expression in vascular walls and moderate on leukocytes, which may be associated with the intensity of neutrophilic infiltration in dermal papillae. Increased serum E-selectin levels indicate the activation of endothelial cells in the development of skin lesions [38].

The important role of IL-8, a granulocyte-macrophage colony-stimulating factor (GM-CSF) and a tumour necrosis factor $\alpha$ (TNF- $\alpha)$ in DH inflammation is also highlighted [40, 41]. Serum level of interleukin 8 (IL-8) is el- 
evated, and its production in the intestine is provoked by gluten, because patients treated with DPS still have high levels, and GFD leads to its lowering [40]. In DH, factors such as trauma or UVB can also induce this cytokine production. Increased IL-8 expression in the basal epidermal layer has a chemotactic effect on neutrophils [42]. The production of GM-CSF, which is able to induce IgA receptor expression by neutrophils at sites of IgA deposits, begins mainly, through dermal-epidermal junction (DEJ). However, this does not explain why these deposits are also found in non-affected skin [9]. The serum of DH patients has higher levels of IL-17a, IL-36 and IL-17 than that of healthy people. IL-17 may also play a role in the activation and recruitment of neutrophils, contributing to tissue damage. IL-36 plays an important role in the recruitment of eosinophils and neutrophils, and stimulates tissue damage and its serum level can be helpful in diagnosing and monitoring disease activity [43-45].

A neutrophil infiltrate is considered to be crucial in the blister formation. Under the influence of collagenase and elastase produced by neutrophils, the basement membrane is damaged and vesicles are formed [17]. After gluten intake within asymptomatic skin, basal keratinocytes begin the production of urokinase-type plasminogen activator stimulating local keratinocytes and macrophages to form MMPs (collagenase and stromiezyzin-1), which then damage the intracellular matrix [46]. The activity of these enzymes, which take part in the physiological and pathological processes of rebuilding extracellular matrix components and in remodelling, is precisely regulated by their tissue inhibitors. The DH imbalance connected with an increased MMP expression and a decrease of their inhibitors, contributes to the formation of vesicles within $24 \mathrm{~h}$ of the stimulus. This is a result of the degradation of major parts of the basement membrane integrity, such as collagen type IV, VII and laminin-1. There is also laminin-5, responsible for the adhesion of keratinocytes to the basal lamina, which reflects local regeneration processes [47].

Mast cells, a source of many mediators, cytokines and enzymes, also play a role in the formation of lesions in $\mathrm{DH}$. As a result of their degranulation, histamine, TNF- $\alpha$ and cytokines are released (IL-1, IL-6 and IL-8). Cell adhesion molecules, chemokines, leukotrienes, platelet activating factor and heparin derivatives also participate in the pathogenesis. Elevated levels of TNF- $\alpha$ and MMP9 are found in bulla fluid, skin lesions, and in perilesional skin $[41,48]$. In the active DH phase, there are elevated levels of circulating antibodies to heat shock proteins (Hsp): Hsp60, Hsp70, Hsp90, which correlate with antibodies against tTg and eTg, and their titre decreases during remission and on GFD. Although their role in the $\mathrm{DH}$ pathogenesis is unknown, they may be a new marker of the disease [49]. There is a significantly higher level of IL-12 in serum and elevated level in perilesional and skin lesions, but the role is unclear. In DH, significantly increased expression of corticoliberin and endothelin B receptor has been demonstrated, indicating their possible involvement in skin pruritus [48]. Studies on pruritus in $\mathrm{DH}$ also point to the role of IL-31, whose serum concentration is significantly lower than in the control group, which may be correlated with its role in the Janusactivated kinases/ signal transducers and activators of transcription (JAK/STAT) signalling pathway [50]. A recent study demonstrated that IL-31 is elevated in DH serum and significantly overexpressed in the skin, where it colocalized with IL31RA [51].

JAK3 is the only protein from the JAK group whose expression is higher in $\mathrm{DH}$ skin lesions than in healthy people. It probably mediates in response to IL-4 and Th17 differentiation. The expression of JAK/STAT proteins in $\mathrm{DH}$ and their suggested role in pathogenesis creates new potential therapeutic targets [52].

Immunohistochemical examination of skin biopsy of $\mathrm{DH}$ patients shows an elevated concentration of B granzyme within DEJ compared to the skin of healthy people. Its presence leads to the degradation of key parts of this combination ( $\alpha 6 / \beta 4$ integrin, collagen VII and collagen XVII) which are substrates for this protease, inducing proteolysis and DEJ separation. It is also found in micro-abscesses, mainly in the upper layer of papillary skin adjacent to DEJ, which suggests the involvement of neutrophils and lymphocytes in the secretion of this enzyme [53].

A protease inhibitor elafin plays an immunomodulatory and regulatory role in epithelium and, among others, inactivates neutrophil elastases and plays a protective role by slowing the gliadin deamidation process in the intestine. Keratinocytes, in normal conditions, show elafin overexpression, thus reducing the inflammatory response induced by neutrophils. In the active DH phase, in the skin, as well as in the intestine in CD, there is abnormal - reduced (in 30\% of patients) or absent (in 70\% of them) expression of elafin, which may be of significant importance in the pathogenesis of these diseases [54].

Dermatitis herpetiformis genetic predisposition, environmental factors and immunological phenomena are important in the development of disease symptoms. Epidermal transglutaminase and neutrophils play a key role in the pathogenesis of this disease. Binding IgA-eTg complex to the dermal papillae stimulates neutrophil influx, proinflammatory cytokine and chemokine release, and metalloproteinase overproduction. Gluten-free diet reduces the development of immune processes.

\section{Conflict of interest}

The authors declare no conflict of interest.

\section{References}

1. Dmochowski M. Autoimmunizacyjne dermatozy pęcherzowe. UM Poznań 2006; 281-311. 
2. Żebrowska A, Waszczykowska E, Kowalewski C, et al. Diagnostyka i postępowanie terapeutyczne w opryszczkowatym zapaleniu skóry (w chorobie Duhringa) - Konsensus Polskiego Towarzystwa dermatologicznego. Dermatol Rev 2016; 103: 95-101.

3. Sun Y, Lin Y, Yang B, et al. The HLA alleles $B^{*} 0801$ and $\mathrm{dRB1}{ }^{*} 0301$ are associated with dermatitis herpetiformis in a Chinese population. J Invest Dermatol 2016; 136: 530-2.

4. Sachs J, Awad J, McCloskey D, et al. Different HLA associated gene combinations contribute to susceptibility for coeliac disease and dermatitis herpetiformis. Gut 1986; 27: 515-20.

5. Katz S, Hertz K, Rogentine G, et al. HLA-B8 and dermatitis herpetiformis in patients with IgA deposits in skin. Arch Dermatol 1977; 113: 155-6.

6. Calonje J, Brenn T, Lazar A, et al. Mckee's Pathology of the skin. $4^{\text {th }}$ edition. Elsevier 2011; 144-7.

7. Antiga E, Caproni M. The diagnosis and treatment of dermatitis herpetiformis. Clin Cosmet Investig Dermatol 2015; 8: 257-65.

8. Ohata C, Ishii N, Hamada T, et al. Distinct characteristics in japanese dermatitis herpetiformis: a review of all 91 Japanese patients over the last 35 years. Clin Dev Immunol 2012; 2012: 562168.

9. Reunala T. Dermatitis herpetiformis. Clin Dermatol 2001; 19: 728-36.

10. Caproni M, Antiga E, Melani L, et al. Italian Group for cutaneous immunopathology. Guidelines for the diagnosis and treatment of dermatitis herpetiformis. J Eur Acad Dermatol Venereol 2009; 23: 633-8.

11. Fry L. Dermatitis herpetiformis: problems, progress and prospects. Eur I Dermatol 2002; 12: 523-31.

12. Dieterich W, Laag E, Bruckner-Tuderman L, et al. Antibodies to tissue transglutaminase as serologic markers in patients with dermatitis herpetiformis. J Invest Dermatol 1999; 113: 133-6.

13. Sárdy M, Kárpáti S, Merkl B, et al. Epidermal transglutaminase (Tgase 3 ) is the autoantigen of dermatitis herpetiformis. J Exp Med 2002; 195: 747-57.

14. Antiga E, Maglie R, Quintarelli L, et al. Dermatitis herpetiformis: novel perspectives. Front Immunol 2019; 10: 1290.

15. Zone J, Meyer L, Petersen M. Deposition of granular IgA relative to clinical lesions in dermatitis herpetiformis. Br J Dermatol 1994; 131: 822-6.

16. Caproni M, Feliciani C, Fuligni A, et al. Th2-like cytokine activity in dermatitis herpetiformis. Br J Dermatol 1998; 138: 242-7.

17. Oikarinen A, Reunala T, Zone J, et al. Proteolytic enzymes in blister fluids from patients with dermatitis herpetiformis. Br J Dermatol 1986; 114: 295-302.

18. Lombardo G, Marabini L, Doneda L, et al. DNA damage in human skin fibroblasts from patients with dermatitis herpetiformis. Eur J Dermatol 2019; 29: 167-73.

19. Oxentenko A, Murray J. Celiac disease and dermatitis herpetiformis: the spectrum of gluten-sensitive enteropathy. Int J Dermatol 2003; 42: 585-7.

20. Makino T, Yoshihisa Y, Mizawa M, et al. Increased serum levels of Th2-type cytokines and eotaxin in fibrillar-type dermatitis herpetiformis. Acta Derm Venereol 2017; 97: 642-3.

21. Savilahti E, Reunala T, Mäki M. Increase of lymphocytes bearing the gamma/delta T cell receptor in the jejunum of patients with dermatitis herpetiformis. Gut 1992; 33: 206-11.

22. Wolnik-Trzeciak G, Bowszyc-Dmochowska M, DańczakPazdrowska A, et al. Budowa i czynności transglutaminaz oraz ich rola w opryszczkowatym zapaleniu skóry. Dermatol Klin 2005; 7: 37-43.

23. Dieterich W, Ehnis T, Bauer M, et al. Identification of tissue transglutaminase as the autoantigen of celiac disease. Nat Med 1997; 3: 797-801.

24. Biagi F, Bassi E, Ardigó M, et al. In patients with dermatitis herpetiformis distribution of transglutaminase in cutaneous tissue does not differ from controls. Dig Liver Dis 2003; 35: 41-5.

25. Clarindo M, Possebon A, Soligo E, et al. Dermatitis herpetiformis: pathophysiology, clinical presentation, diagnosis and treatment. An Bras Dermatol 2014; 89: 865-77.

26. Kárpáti S. Dermatitis herpetiformis: close to unravelling a disease. J Dermatol Sci 2004; 34: 83-90.

27. Kárpáti S, Sárdy M, Németh K, et al. Transglutaminases in autoimmune and inherited skin diseases: the phenomena of epitope spreading and functional compensation. Exp Dermatol 2018; 27: 807-14.

28. Helin H, Mustonen J, Reunala T, et al. IgA nephropathy associated with celiac disease and dermatitis herpetiformis. Arch Pathol Lab Med 1983; 107: 324-7.

29. Kawana S, Segawa A. Confocal laser scanning microscopic and immunoelectron microscopic studies of the anatomical distribution of fibrillar IgA deposits in dermatitis herpetiformis. Arch Dermatol 1993; 129: 456-9.

30. Gornowicz-Porowska J, Seraszek-Jaros A, Bowszyc-Dmochowska M, et al. Immunoexpression of IgA receptors (CD89, CD71) in dermatitis herpetiformis. Folia Histochem Cytobiol 2017; 55: 212-20.

31. Brown L, Harrist T, Yeo K, et al. Increased expression of vascular permeability factor (vascular endothelial growth factor) in bullous pemphigoid, dermatitis herpetiformis, and erythema multiforme. J Invest Dermatol 1995; 104: 744-9.

32. Preisz K, Sárdy M, Horváth A, et al. Immunoglobulin, complement and epidermal transglutaminase deposition in the cutaneous vessels in dermatitis herpetiformis. J Eur Acad Dermatol Venereol 2005; 19: 74-9.

33. Provost T, Tomasi T. Evidence for the activation of complement via the alternate pathway in skin diseases. II. Dermatitis herpetiformis. Clin Immunol Immunopathol 1974; 3: 178-86.

34. Seah PP, Fry L, Mazaheri MR, et al. Alternate-pathway complement fixation by IgA in the skin in dermatitis herpetiformis. Lancet 1973; 2: 175-7.

35. Katz S, Strober W. The pathogenesis of dermatitis herpetiformis. J Invest Dermatol 1978; 70: 63-75.

36. Dahlbäck K, Löfberg H, Dahlbäck B. Vitronectin colocalizes with Ig deposits and C9 neoantigen in discoid lupus erythematosus and dermatitis herpetiformis, but not in bullous pemphigoid. Br J Dermatol 1989; 120: 725-33.

37. Haffenden G, Blenkinsopp W, Ring N, et al. The potassium iodide patch test in the dermatitis herpetiformis in relation to treatment with a gluten-free diet and dapsone. Br J Dermatol 1980; 103: 313-7.

38. Hall R. The pathogenesis of dermatitis herpetiformis: recent advances. J Am Acad Dermatol 1987; 16: 1129-44.

39. Smith A, Streilein R, Hall R. Neutrophil CD11b, L-selectin and FC IgA receptors in patients with dermatitis herpetiformis. Br J Dermatol 2002; 147: 1109-17.

40. Hall R, Takeuchi F, Benbenisty K, et al. Cutaneous endothelial cell activation in normal skin of patients with dermatitis herpetiformis associated with increased serum levels of IL-8, sE-Selectin, and TNF-alpha. J Invest Dermatol 2006; 126: $1331-7$. 
41. Żebrowska A, Wągrowska-Danilewicz M, Danilewicz M, et al. Mediators of mast cells in bullous pemphigoid and dermatitis herpetiformis. Mediators Inflamm 2014; 2014: 936545.

42. Takeuchi F, Stenlein R, Hall R. 3rd Increased E-selectin, IL-8 and IL-10 gene expression in human skin after minimal trauma. Exp Dermatol 2003; 12: 777-83.

43. Żebrowska A, Wagrowska-Danilewicz M, Danilewicz M, et al. IL-17 expression in dermatitis herpetiformis and bullous pemphigoid. Mediators Inflamm 2013; 2013: 967987.

44. Velikova T, Shahid M, Ivanova-Todorova E, et al. Celiacrelated autoantibodies and IL-17a in bulgarian patients with dermatitis herpetiformis: a cross-sectional study. Medicina 2019; 55: 136.

45. Żebrowska A, Woźniacka A, Juczyńska K, et al. Correlation between IL36 $\alpha$ and IL17 and activity of the disease in selected autoimmune blistering diseases. Mediators Inflamm 2017; 2017: 8980534.

46. Airola K, Reunala T, Salo S, et al. Urokinase plazminogen activator is expressed by basal keratinocytes before interstitia collagenase, stromelysin-1, and laminin- 5 in experimentally induced dermatitis herpetiformis lesions. J Invest Dermatol 1997; 108: 7-11.

47. Żebrowska A, Narbutt J, Sysa-Jędrzejowska A, et al. The imbalance between metalloproteinases and their tissue inhibitors is involved in the pathogenesis of dermatitis herpetiformis. Mediators Inflamm 2005; 6: 373-9

48. Cynkier A, Żebrowska A, Wągrowska-Danilewicz M, et al. Expression of selected neuropeptides in pathogenesis of bullous pemphigoid and dermatitis herpetiformis. Pol I Pathol 2012; 63: 31-9.

49. Kasperkiewicz M, Tukaj S, Gembicki A, et al. Evidence for a role of autoantibodies to heat shock protein 60,70 , and 90 in patients with dermatitis herpetiformis. Cell Stress Chaperon 2014; 19: 837-43.

50. Kulczycka-Siennicka L, Cynkier A, Waszczykowska E, et al. The role of interleukin-31 in pathogenesis of itch and its intensity in a course of bullous pemphigoid and dermatitis herpetiformis. Biomed Res Int 2017; 2017: 5965492.

51. Bonciani D, Quintarelli L, Del Bianco E, et al. Serum levels and tissue expression of interleukin-31 in dermatitis herpetiformis and bullous pemphigoid. J Dermatol Sci 2017; 87: 210-2.

52. Juczyńska K, Woźniacka A, Waszczykowska E, et al. Expression of the JAK/STAT signaling pathway in bullous pemphigoid and dermatitis herpetiformis. Mediators Inflamm 2017; 2017: 6716419.

53. Russo V, Klein T, Lim D, et al. Granzyme B is elevated in autoimmune blistering diseases and cleaves key anchoring proteins of the dermal-epidermal junction. Sci Rep 2018; 8: 9690.

54. Ollague J, Nousari C. Expression of Elafin in Dermatitis Herpetiformis. Am J Dermatopathol 2018; 40: 1-6. 